\title{
PHYTOPATHOLOGIE ET PRODUCTION LIGNEUSE
}

\author{
L. LANIER \\ Laboratoire de Pathologie forestière, \\ Centre national de Recherches forestières, I. N. R. A., \\ Champenoux 54370 Einville
}

\begin{abstract}
RÉSUMÉ
La phytopathologie forestière, qui se préoccupe de l'étude des maladies des arbres forestiers et des moyens de lutter contre elles, a besoin, en particulier pour définir et classer par ordre d'urgence les programmes de recherche, d'une estimation chiffrée des dégâts en forêt. Certaines enquêtes américaines ont montré que le pourcentage des pertes pouvait être considérable ( $48 \mathrm{p}$. 100 de la production annuelle aux U. S. A.). Il faut préciser que beaucoup de peuplements forestiers d'Amérique du Nord ne sont pas soumis, comme en Europe, à une sylviculture intensive. Dans nos régions toutefois et pour certaines essences, les estimations de pertes peuvent également justifier une intervention et en tous cas une intensification des efforts de recherches.

Celles-ci porteront sur les effets des facteurs physico-chimiques, climatiques et biotiques, influant à la fois sur le comportement des agents phytopathogènes et sur la résistance plus ou moins bien adaptée des hôtes. Les études sont complexes, du fait des nombreuses interactions qui s'exercent entre les différents facteurs en cause; mais les résultats dès à présent obtenus, l'amélioration de nos connaissances sur la biologie des parasites et des arbres permettent de lutter souvent efficacement, même si les solutions actuellement retenues sont encore loin d'ŝtre parfaites.
\end{abstract}

\section{INTRODUCTION}

Les facteurs fondamentaux qui gouvernent la production ligneuse sont :

- le choix d'une espèce ou d'une variété productive (sous-entendu adaptée à la station),

- une sylviculture optimisée comportant l'amélioration (si nécessaire et si possible) des conditions de sol,

- la santé des arbres.

C'est en principe à ce dernier niveau seul qu'intervient la phytopathologie forestière, qui est l'étude de la nature et du développement des maladies chez les arbres, ainsi que des moyens de lutte opposables. 
Ce qui complique l'analyse des causes des maladies, c'est d'une part la nature particulière du végétal en cause, l'arbre, et d'autre part le nombre des interactions qui s'exercent entre l'hôte, son ou ses parasites et les facteurs écologiques.

Pour tenter de simplifier cette étude et après un bref rappel des caractéristiques particulières de notre hôte, nous verrons successivement :

1. Influence des champignons phytopathogènes sur la production ligneuse.

2. Effets des facteurs écologiques sur les champignons phytopathogènes.

Caractéristiques de l'arbre, propres à expliquer les particularités de la pathologie forestière.

L'arbre, avec sa partie ligneuse, possède des caractérisques qui le différencient nettement de la majorité des autres végétaux. Les contraintes auxquelles il est soumis, en plus de la concurrence de ses voisins, concernent l'exposition permanente aux risques météorologiques : la foudre, le vent, la grêle, le poids de la neige, et le visent d'autant plus qu'il émerge.

Il se différencie plus nettement encore des autres végétaux par son caractère pluriannuel qui l'expose, au fil des années, aux attaques de ses ennemis végétaux ou animaux dont le cycle biologique est, lui, généralement saisonnier.

Pour saisir le rôle exact de ces éléments du milieu qui concourent à la décomposition de la matière ligneuse, il faut avoir parcouru l'une de ces forêts vierges où, bien évidemment, la production ligneuse (au sens d'exportation par les coupes) est exactement nulle puisque équilibrée par la destruction. Ce ne sont que troncs morts enchevêtrés, arbres dressant vers le ciel des branches mortes qui s'effondrent au moindre choc.

Il n'y a plus de ces forêts naturelles en France et nos réserves dites artitisques ou les forêts de protection à la limite de végétation en montagne n'en sont que de pâles images. Ces réserves nous aident toutefois à comprendre ce fait essentiel : le prélèvement que, grâce à la sylviculture, nous effectuons aux dépens des forêts et qui s'appelle production ligneuse l'est au détriment des êtres vivants qui décomposent la matière morte qu'est le bois pour en faire de l'humus.

Ces êtres vivants ne sont pas supprimés pour autant dans une forêt aménagée et l'homme entrant en concurrence avec eux pour son prélèvement de bois doit s'intéresser à leur biologie afin d'en minimiser au mieux les effets.

\section{I. - INFLUENCE DES CHAMPIGNONS PHYTOPATHOGÈNES SUR LA PRODUCTION LIGNEUSE}

\section{1. - Généralités}

L'effet des maladies cryptogamiques sur les arbres est divers : elles peuvent entrainer la mort de l'arbre, la destruction du bois, des réductions de croissance, une diminution des qualités technologiques, différer des régénérations, altérer le bois stocké, modifier la composition des forêts en l'orientant vers des peuplements de moindre valeur ou dégrader le paysage forestier. L'ordre de ces différents effets est indiqué ici en gros par importance économique décroissante. 
Le problème posé par l'estimation de ces effets est d'une importance primordiale à la fois pour l'économie forestière et pour la recherche en pathologie, puisque sa solution devrait constituer en principe le préalable à tout choix dans les priorités à accorder aux programmes de recherches. Il est loin d'être résolu de nos jours.

La solution dépend d'une coordination des efforts entre services d'inventaire de la production, méthodologistes biométriciens et phytopathologistes. Or, rares sont les régions du monde où ces trois services sont suffisamment développés pour étendre leurs investigations communes à l'ensemble de la production forestière.

On se contente le plus souvent d'approximations, obtenues à l'aide de méthodes de calcul variées ou d'extrapolations à partir d'échantillonnages également très variés. On conçoit dans ces conditions que des divergences gênantes apparaissent, même lorsque l'on s'intéresse aux dégâts causés par un seul champignon sur une même espèce dans des contrées écologiquement voisines.

Il est d'autant plus difficile d'établir une doctrine sérieuse à ce sujet qu'on sait dès à présent les difficultés que l'on va rencontrer :

1. Il est pratiquement impossible de définir un type de mesure homogène valable pour un ensemble de parasites et dans tous les pays.

2. Une perte de production ne sera qu'exceptionnellement due à un seul parasite. Elle résultera le plus souvent des pertes cumulées sous l'effet de plusieurs agents pathogènes, agissant conjointement ou successivement.

3. Les pertes varient considérablement d'une région à une autre en fonction des facteurs écologiques (voir deuxième partie).

4. Il n'y a non pas addition mais combinaison souvent complexe de l'influence des parasites pris un à un.

Soit P la perte cumulée par les pertes élémentaires

$$
p_{1}+p_{2}+\ldots+p_{n}
$$

Si $p_{1}$ pour diverses raisons (écologiques ou tenant à l'hôte) devient $p^{\prime}{ }_{1}$, on aura une nouvelle perte cumulée

$$
\mathbf{P}^{\prime}=p_{1}^{\prime}+p_{2}^{\prime}+\ldots+p_{n}^{\prime},
$$

différente de

$$
p^{\prime}{ }_{1}+p_{2}+\ldots+p_{\mathrm{n}}
$$

Malgré tout, des tentatives plus ou moins ambitieuses ont fait l'objet de publications ces dernières années. Nous avons sélectionné quelques exemples particulièrement significatifs.

\section{2. - Amérique du Nord}

L'Amérique du Nord est le pays des vastes enquêtes et de l'audace dans les conclusions et leurs conséquences.

Entre 1954 et 1958 a été établi un rapport général sur les ressources en bois et les pertes dues à divers agents. D'après les auteurs de l'enquête, les dégâts indiqués sont sous-estimés (Davidson et Buchanan 1964). 


\begin{tabular}{|c|c|c|}
\hline $\begin{array}{l}\text { Ressources en bois } \ldots \ldots \ldots \ldots \\
\text { (U.S.A. y compris Alaska) } \ldots \ldots \\
\text { Croissance annuelle (estimée) } \ldots \ldots \\
\text { Exploitations en } 1962 \ldots \ldots \ldots \ldots\end{array}$ & $\begin{array}{l}605 \text { milliards de pieds-cubes } \\
\text { sur } 489 \text { millions d'acres } \\
14,2 \text { milliards de pieds-cubes } \\
10,2 \text { milliards de pieds-cubes }\end{array}$ & $\begin{array}{l}\text { env. } 20 \text { milliards de mètres-cubes } \\
\text { env. } 210 \text { millions d'hectares } \\
\text { env. } 400 \text { millions de mètres-cubes } \\
\text { env. } 300 \text { millions de mètres-cubes }\end{array}$ \\
\hline
\end{tabular}

Pertes estimées (par l'effet des principales maladies)

(en millions de pieds-cubes)

1. - Pourridiés (sur Douglas surtout)

U.S.A.

115

CANADA

y compris Fomes annosus ( 8 millions de pieds-cubes à lui seul).

2. - Tige et tronc

Pourritures du cœur

Rouille vésiculeuse des

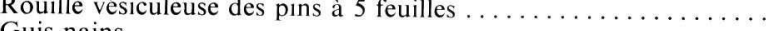

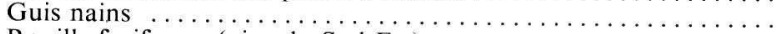

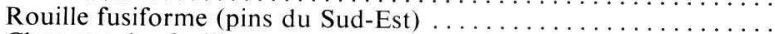

Chancres des feuillus $\ldots \ldots \ldots \ldots \ldots \ldots \ldots$

3. - Maladies foliaires

Taches brunes des aiguilles

Elytroderma (cf. Lophodermium)

4. - Maladies systémiques

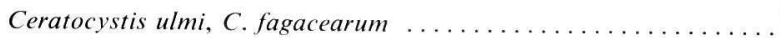

5. - Divers

\begin{tabular}{|c|c|c|}
\hline & 700 & 12 \\
\hline 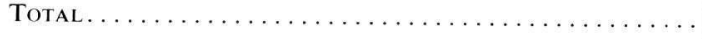 & 4943 & 958 \\
\hline
\end{tabular}

Ce qui représente au total 48 p. 100 aux U.S.A. et 28 p. 100 au Canada de la coupe annuelle*. Si l'on compare les pertes dues aux maladies avec les dégâts ayant d'autres causes pour origine, on constate que les maladies occupent le premier rang parmi les causes de destruction des produits forestiers. Les insectes tuent davantage d'arbres, mais l'incidence des maladies sur l'accroissement total, en particulier par suite des dégâts dûs aux pourritures du cœur, dépasse celle de tout autre agent.

En Amérique du Nord, l'importance des pourritures tient essentiellement à deux causes :

- l'abondance des peuplements surannés et l'on sait que le pourcentage de pourritures, lié à la pénétration par blessures, augmente avec l'âge,

- le fait que de nombreux peuplements feuillus de l'Est des U.S.A. proviennent de régénérations sur souches, toujours plus sensibles aux pourritures du pied.

* Il existe aux U.S.A. et au Canada de nombreux peuplements exagérément vieillis non soumis à exploitation régulière : les pourcentages indiqués doivent donc être comparés avec précaution aux chiffres européens qui vont suivre. 
En conclusion, on peut dire que les chiffres indiqués, bien que largement extrapolés, ont le mérite essentiel d'établir des ordres de grandeur utilisables sur le plan économique et de fournir des critères de choix parmi les recherches à entreprendre.

\section{3. - Europe}

De tels chiffres n'existent pas pour l'Europe. Plus prudents ou plus objectifs, en tous cas beaucoup moins bien équipés dans le domaine des études économiques à grande échelle, nous en savons beaucoup moins sur l'impact réel des maladies sur la production forestière.

Au Congrès I.U.F.R.O. de 1964, ZYCHA présentait un bilan assez prudent, compte tenu des multiples difficultés rencontrées lors de son établissement.

Même lorsque l'on s'intéresse aux dégâts causés par un seul agent, on est frappé par les divergences dans les estimations selon les pays, probablement par suite des différences de méthodologie.

Ainsi pour Fomes annosus, qui est en Europe l'un des agents parasitaires dont l'importance économique est la plus grande, on relève les chiffres suivants :

En Belgique, on considère que 100000 ha de forêts, soit 17 p. 100 de la surface boisée, sont menacés par ce champignon.

En Pologne, 20000 ha seraient partiellement atteints (OrLOs et DoMINIK 1960).

Un inventaire portant sur l'ensemble des pessières du Sud de la Finlande a montré que 10 à 12 p. 100 de tous les épicéas étaient attaqués à des degrés divers (KANGAS 1952).

Sur le plan quantitatif et économique, il est plus intéressant de chiffrer la proportion de bois pourri par rapport à la récolte. En Norvège, la perte annuelle par rapport à la production totale d'épicéa est de $200000 \mathrm{~m}^{3}$ soit environ 3,3 p. 100. En Suède (Rennerfelt 1957), une estimation de ce même rapport montait à 14 p. 100, tandis qu'au Danemark, HolmsGAARD (1957) arrivait à 6,6 p. 100.

Si l'on fait intervenir pour ces estimations non plus le pourcentage d'arbres atteints, mais la perte économique en valeur, on arrive encore à des éventails de chiffres plus larges, étant donné qu'en Allemagne en particulier, une proportion importante de bois pourri reste utilisée pour la fabrication des pâtes.

D'une étude récente et relativement complète conduite en Basse-Saxe par ZYCHA et КАтO (1967), on peut tirer quantité de renseignements chiffrés très intéressants par leur précision.

TABLEAU

\begin{tabular}{|c|c|c|c|c|}
\hline \multirow[b]{2}{*}{ Placettes } & \multicolumn{3}{|c|}{ Enquête Meyer et Brenken 1960} & \multirow{2}{*}{ Enquête DoBele 1962} \\
\hline & A & B & $\mathrm{C}$ & \\
\hline Pourcentage de bois pourri............ & 17,7 & 20,0 & 26,0 & 28,3 \\
\hline Réduction de valeur (en p. cent) ......... & 10,0 & 12,4 & 8,7 & 14 \\
\hline
\end{tabular}


Le pourcentage d'arbres atteints selon les peuplements varie de 7 à 88 p. 100. Le pourcentage de bois pourri pour l'ensemble des abattages d'épicéa en Basse-Saxe est de l'ordre de 20 p. 100. Bien entendu, le pourridié à Fomes ne représente qu'une fraction de ces 20 p. 100 (environ le tiers).

On peut considérer ce travail de ZYCHA et KATO, d'autant plus intéressant pour nous qu'il concerne un parasite et une espèce-hôte bien représentés en France et dans des conditions comparables à celles de la Basse-Saxe, comme l'une des meilleures contributions européennes à l'étude de l'influence d'un parasite cryptogamique sur une production forestière.

Aux effets économiques étudiés, s'ajoutent des tentatives de corrélations entre l'importance des dégâts et certains facteurs écologiques, les plus importants semblant l'altitude, l'orientation de la pente et le $\mathrm{pH}$ du sol.

Nous tentons actuellement de mettre au point une méthode d'estimation des dégâts dûs au Fomes sur l'Épicéa, qui prolongera les travaux allemands dans le cadre des plateaux du Nord-Est de la France. Nous y serons largement aidés par le réseau de placettes d'observations installées par nos collègues sylviculteurs et pédologues, et, sans vouloir minimiser l'intérêt de leurs récentes études, nous espérons pouvoir nuancer les chiffres de production probable auxquels ils sont arrivés, en introduisant le facteur correctif dû à l'effet du Fomes.

Dans le même ordre d'idées, on peut signaler encore les travaux italiens récents sur Marssonina brunnea. Étant donné qu'il s'agit d'un parasite foliaire, agissant pratiquement seul et contre lequel la lutte chimique est bien au point, Cellerino (1969), Castellani et Cellerino (1969) ont comparé simplement la production ligneuse de peupleraies traitées et non traitées, en fonction des clônes.

$\begin{array}{lr}\text { I } 214 & 13 \% \\ \text { I } 488 & 30 \% \\ \text { I } 262 & 30 \% \\ \text { B 2F } & 30 \% \\ \text { I } 455 & 25 \% \\ \text { Carolins } & 8 \%\end{array}$

Pour l'ensemble des clônes utilisés en Italie, la perte due au Marssonina est estimée à environ $16 \%$ de la production.

Le cas était particulier et n'était pas sans analogie avec celui étudié récemment par nos collègues sylviculteurs (BOUCHON et Tотн 1971) : évaluation des pertes de production dues aux attaques de la chenille processionnaire sur Pin noir. La perte d'accroissement, observée par l'intermédiaire des variations d'épaisseur des cernes, est considérable. Ceci peut tenir à l'optimisme de l'hypothèse faite par les auteurs sur l'indépendance entre les dégâts de processionnaire et les autres causes de perte de production. Il est certain que l'approche particulièrement intéressante qui a été tentée doit être complétée, pour la même essence et dans des conditions écologiques comparables, par des études d'effeuillages artificiels tels que ceux entrepris par JOLY (1969).

Pour un champignon phytopathogène, parasite foliaire et dont l'effet principal est de faire tomber les aiguilles, nous avons dû pratiquer également ces défoliaisons artificielles toujours fastidieuses et, qui plus est, sujettes à critiques étant donné les effets secondaires qu'elles entraînent (LANIER 1969). 
Des études de ce genre ne pourront véritablement être considérées comme complètes rô probantes que lorsque l'on connaîtra de façon parfaite la composition, la biomasse et le logique posé passe parties constitutives des arbres. La solution du problème phytopatho-

\section{4. - Reste du Monde - Perspectives}

C'est paradoxalement à un stade très avancé de la recherche, lorsque l'on connaît l'essentiel de la biologie d'un agent parasitaire, que l'on se préoccupe de connaître les effets sur l'hôte. Le cas de la Processionnaire est significatif à cet égard.

On rencontre rarement le cadre idéal pour des recherches à la fois biologiques et économiques sur l'effet des parasites en forêt. Pourtant on en est très près avec l'étude du Dothistroma pini sur Pinus radiata en Nouvelle Zélande (GILMOUR et SUTTON 1967) : parasite foliaire quasiment exclusif sur cet hôte, à effet connu, à biologie bien étudiée, contre lequel les moyens de lutte chimique sont également bien au point et chiffrables.

Dans ce cas, unique à notre connaissance en forêt, et exceptionnellement favorable, le pathologiste s'est associé à un économiste et a pu chiffrer très exactement :

- la production en l'absence du parasite, - I'effet du parasite sur la production, en relation avec différents degrés d'attaque
définis selon une échelle précise, d'attaque.

- le coût des traitements et ce qu'on pouvait en espérer (rentabilité) selon le degré

En dehors de ce cas exceptionnel, le travail qui paraît offrir les perspectives les plus prometteuses est celui de FosTer et JOHNSON (1963). Il s'est agi dans une première phase d'estimer les dégâts causés par divers pourridiés et maladies desséchant le Douglas. Dans une seconde phase, les résultats obtenus ont été traités à l'aide de différentes méthodes d'échantillonnage et ces méthodes comparées pour leur précision. Ainsi on peut choisir des méthodes de capture et de traitement des données dans des cas comparables.

On peut donc dire, en conclusion de ce chapitre, que l'impact des champignons phytopathogènes sur la production ligneuse est très imparfaitement connu, et qu'une part importante de nos efforts des prochaines années devra être consacrée à ce problème.

A noter d'ailleurs que l'aide des écologistes, des sylviculteurs s'intéressant à la production, des biométriciens, voire des économistes, est vivement sollicitée pour ce travail.

\section{SUR LES CHAMPIGNONS PHYTOPATHO ÉCOLOGIQUES}

Il est des cas simples où cet effet va de soi : par exemple la présence ou l'absence d'une espèce végétale en association avec une autre explique ou exclut une attaque de Rouille lorsque celle-ci nécessite, pour boucler son cycle biologique, l'alternance sur les deux espèces.

Mais autant, si l'on considère chaque facteur écologique pris isolément, l'étude de ses 
effets sur nos champignons est relativement aisée *, autant elle devient malaisée lorsque l'on souhaite approfondir nos connaissances en rapprochant le dispositif des conditions réelles, c'est-à-dire si l'on souhaite expliquer les interactions.

\section{1. - Facteurs physico-chimiques}

Les facteurs physico-chimiques du milieu dont on a généralement à envisager l'action sont :

- dans l'air : sa température, son état hygrométrique, sa composition, y compris les gaz délétères qu'il peut contenir, la fréquence et l'intensité des précipitations;

— dans le sol parallèlement : sa température, son état hydrique, son $\mathrm{pH}$ et sa fertilité.

Ce dernier facteur dépend déjà pour une part des agents biologiques qui concourent à modifier cette fertilité et permet la transition avec les facteurs biotiques du milieu dont l'influence est souvent prépondérante.

Quelques exemples nous éclaireront mieux sur les effets de ces facteurs physicochimiques.

La majorité des champignons phytopathogènes ne peuvent se développer efficacement que dans une gamme de températures bien précises. Les températures élevées, à partir de $30^{\circ}$ pour nos parasites les plus courants, sont létales pour le mycélium, les températures très basses inhibent leur développement. C'est ainsi que le Phacidium infestans Karst., parasite des aiguilles des pins et du Pin sylvestre en particulier, n'est dommageable que dans les régions les plus septentrionales de l'Europe et que le Lophodermium pinastri (Schrad.) Chev., autre parasite aciculaire des pins, est pratiquement inconnu sur les pins des régions intertropicales.

L'état hygrométrique de l'air commande fréquemment le mécanisme de l'infection. Nous avons pu montrer que la fréquence des étés secs dans certaines régions d'Amérique du Nord était en étroite corrélation avec la relative inocuité du Lophodermium pinastri. Inversement, DURRIEU (1957) a expliqué par un état hygrométrique généralement plus élevé dans sa zone d'introduction européenne que dans sa zone d'origine américaine, que le pouvoir infectieux du Phaocryptopus gauemanni était renforcé sur les Douglas implantés dans nos régions.

Dans les sols, certaines caractéristiques biologiques propres à des parasites comme les Phycomycètes du type Pythium ou Phytophthora par exemple, dont le cycle comporte une phase sous forme de zoospores nageuses, expliquent que les sols mouilleux ou des irrigations favorisent la dissémination et l'extension des maladies causées par ces Phycomycètes.

L'acidité et la plus ou moins grande richesse des sols en éléments minéraux justifient également le comportement et l'abondance de parasites exigeants à ces points de vue.

Mais déjà, pour ce qui est de ces facteurs et plus encore quand il s'agira de fertilité, interfère la présence d'autres organismes vivants qui viennent renforcer ou diminuer l'incidence des pathogènes.

* Il suffit de disposer du matériel expérimental permettant de faire varier ce facteur seul. 


\section{2. - Facteurs biotiques}

Rares sont les milieux naturels abiotiques et le milieu forestier l'est certainement moins que tout autre.

Le parasitisme d'un champignon ne peut s'exercer que dans un contexte où interviennent :

- d'autres champignons,

- des bactéries, des virus,

- les insectes, les nématodes, d'autres animaux.

Là encore, on peut citer quelques exemples au milieu d'un foisonnement d'études qui font l'objet d'une part importante des recherches en phytopathologie, sans doute la plus profitable à terme.

Vis-à-vis des autres champignons, trois types de comportement des champignons phytopathogènes ont pu être définis :

- l'indifférence, qui est rare,

- l'opposition, qui est la règle générale, avec ses sous-types : simple concurrence pour la colonisation du milieu, inhibition limitée ou antibiose franche. Les travaux actuels de Delatour tendent à exploiter ces phénomènes naturels à des fins pratiques pour lutter contre la pourrituie rouge du cœur de l'Épicéa.

- l'association ou la succession sur un même milieu, chaque associé apportant à son commensal des conditions plus favorables à son développement. Les ligninolytiques du bois s'associent ainsi avec les cellulolytiques pour accélérer l'élaboration de cette substance relativement stable qu'est l'humus.

Les bactéries et les virus de même agissent soit en affaiblissant l'arbre et en facilitant ainsi sa destruction par des saprophytes à tendance parasitaire, soit - avec certitude pour les virus - en affaiblissant le champignon parasite et en offrant ainsi les moyens d'une lutte biologique originale. Les récents travaux de GrENTE (1969) sur les races hypovirulentes de l'Endothia parasitica, agent du chancre du Châtaignier, laissent supposer l'existence possible de viroses ou du moins de phénomènes naturels susceptibles de limiter la virulence chez de nombreux champignons phytopathogènes.

Quant aux insectes, aux nématodes et aux autres animaux, ils jouent parfois un rôle fondamental. Les insectes peuvent se nourrir de champignons, les nématodes les détruire et réciproquement, tous peuvent les véhiculer, donnant ainsi des exemples d'équilibre biologique, de biocœnoses complexes ou au contraire de symparasitisme parfois très étroit, comme dans le cas de la maladie de l'Orme liée aux transports par Scolytes par exemple.

A partir du moment où s'établissent des liens entre plusieurs êtres vivants, y compris l'hôte et l'un de ses parasites, on peut parler d'interactions, et, dans ce domaine, comme disait PASCAL « l'imagination de l'homme se lassera plutôt de concevoir que la nature de fournir ».

Un exemple d'interaction typique et complexe est constitué par l'association mycorrhizienne, un peu mieux connue maintenant. Des exemples devenus classiques montrent qu'un 
plant mycorrhizé est moins sensible aux parasites cryptogamiques racinaires qu'un plant non mycorrhizé.

L'explication vient sans doute d'être trouvée grâce aux travaux de KRUPA (1972) qui, en Finlande, vient de démontrer que des composés volatils appartenant aux groupes des terpènes étaient émis à proximité des racines de Pin mycorrhizées par le Boletus variegatus. Ces produits inhibent in vitro la croissance des parasites Phytophthora cinnamomi et Fomes annosus.

\section{CONCLUSIONS}

On pourrait multiplier à l'envi ce genre d'exemples.

La Pathologie forestière, en tant qu'étude des maladies des arbres, ne représente somme toute que la recherche des causes d'un certain déséquilibre affectant des mécanismes qui devraient normalement travailler au profit de l'homme et qui, brusquement ou insensiblement, ne le font plus ou le font moins bien.

C'est pourtant dans cette discipline peut-être que les difficultés rencontrées dans le démontage du jeu physiologique sont les plus sensibles, car si l'arbre est dépourvu de ce « milieu intérieur » qu'a défini Claude BERNARD en physiologie animale et dont la stabilité sert de repère utile au médecin, il est d'autant plus sensible aux influences extérieures et les ressent en se modifiant s'il le peut, en étant malade s'il ne le peut pas.

Comme en définitive toute modification, si discrète soit-elle, retentira sur la physiologie de l'arbre, donc sur sa production, on peut conclure que toute recherche approfondie en pathologie passera par de meilleures connaissances sur le fonctionnement de ce matériel vivant original qu'est l'arbre.

\section{SUMMARY}

\section{PHYTOPATHOLOGY AND WOOD PRODUCTION}

To define and classify the research programs in forest pathology according to their importance, it is necessary to know the importance of the damages on wood production.

According to certain publications from the United States, the loss can be as high as 48 p. 100 of the annual wood production. It should be emphasized however, that most of the american forests are not as intensively managed as in the european forests. But, in spite of this, wood production loss might justify in certain areas and for certain species more intensive research in pathology.

Physico chemical, climatical on biological factors having an influence both on pathogenic agents and on the more or less resistant and adapted host should be studied.

This is complicated due to the many interactions between the different factors involved. But the results obtained, and the improvements of our knowledge on the biology of parasites and hosts allow the use already effective means of control with success although the solutions used at present are far from being perfect.

\section{RÉFÉRENCES BIBLIOGRAPHIQUES}

Anonyme, 1958. - Timber resources for America's future. Forest Resource Report no ${ }^{14}$, Forest Service U.S.D.A.

Anonyme, 1965. - Importance des maladies et des insectes nuisibles à la forêt. Unasylva, 19, 3, $\mathrm{n}^{\circ} 78$, 99-102. 
Bouchon J., Tотн J., 1971. - Étude préliminaire sur les pertes de production des pinèdes soumises aux 323-340.

Castellani E., Cellerino G. P., 1969. - Valutazione delle perdite in dotte dalla nella produzione legnosa del pioppo nell' Italia de perdite in dotte dalla Marssonina brunnea

Cellerino G. P., 1969. - Pratica applicazione della in Italia. Cellulosa e Carta, 12, 25-31.

Davidison Q. G., Buchanan T. S., 1964

I.U.F.R.O. Symposium, Meeting $n^{\circ}$ VI. )

Dobele E., 1962. - Betriebswirtschaftiche Unters

88, 1862-1864, 1873-1876.

Durrieu G., 1957. - Influence du parasite du Pseudotsuga. C. R. Acad. Sci., 244, 2183-2185.

Foster R. E., Johnson A. L. S., 1963. - Assessmen ts 2183-2185. forest disease in Douglas fir plantations. Canada, 5-52.

Gilmour J. W., Sutton W. R. J., 1967 . and New Zealand. F.A.O. I.U.F.R.O. Antroduction to quantification of disease losses in Australia

Grente J., 1969. - L'hypovirulence exclusive Symposium. Munchen, Sect. 24. C.R. Acad. Sci., Série D, 268, 3173-3176.

Grente J., 1969. - L'hypovirulence exclusive, phé Sci., Série D, 268, 2347-2350. Dansk. Skovforen. Tidsskr., 42, 237-343.

JOLY R., 1969 — Incidence économique des insecte riences d'effeuillage artificiel. C.R. hebdo. Séancesêt. Quelques chiffres intéressants sur des expé-

Kangas E., 1952. - On the incidence, infection and in Finland. Commun. Inst. For. Fenn., 40, 259-274.

KRUPA H., 1972. - Recherches sur les ectomycorrhizes champignons parasites des racines par des composés or. III - Inhibition de croissance de deux racinaire mycorrhizé du Pinus silvestris L. Eur.

Lanier L., 1969. - Contribution à l'étude du Roun silvestris L. Eur. J. For. Path., II (sous presse). pinastri (Schrad.) Chev. Thèse, Nancy, $177 \mathrm{p}$.

Meyer-Brenken H., 1960. - Begriff und Aushaltung o

86, 801-802.

Orlos H, Dominik T., 1960. - On the biology of the root fungus F. annosus. Sylwan, 104, 13. RENnERFElt E., 1957. - Untersuchungen über die Wurzelfäule auf Fichte und Kiefer in Schweden. Phy-
topathol. Z., 28, 259-274.

ZYCHA H., 1964. - Report on forest diseases situation in Europe with emphasis on the impact on forest production. F.A.O./I.U.F.R.O. Symposium Meeting $n^{\circ}$ VI.

ZYCHA H., KaTO F
39, $117 \mathrm{p}$.

\section{DISCUSSION}

Au cours de la discussion qui s'instaure à la suite de l'exposé LANIER, les principales questions posées et les réponses apportées ont été les suivantes.

M. l'Ingénieur Général Pourtet :

1. - Une distinction doit être faite entre les pertes enregistrées en Amérique du Nord dans les forêts vierges et non soignées et celles causées par de véritables maladies, telle par
exemple la Rouille vésiculeuse des Pins à cinq feuilles.

2. - On peut donner un exemple de l'effet d'un facteur physique et d'une interaction à propos du Chancre bactérien du Peuplier dont l'agent causal a été identifié par RidE 
- la bactérie ne peut se développer au-dessus d'une certaine température, d'où l'absence de ses dégâts au Sud de Paris;

- un Diptère du genre Dendromyza est par ses déplacements dans ses galeries un véhicule de la bactérie.

LANiER remercie M. Pourtet des compléments d'information ainsi apportés. Bien entendu, quantité d'autres exemples auraient pu être cités de ces types d'actions et d'interactions des facteurs écologiques en pathologie, mais ils auraient largement débordé le temps limité qui nous était imparti.

M. TIMBAL, à propos d'une remarque préliminaire de M. LANIER, qui ne voyait figurer nulle part sur le tableau général de la biocœnose forestière, les champignons phytopathogènes des forêts, précise que leur flore fait bien évidemment partie de cette biocœnose et plus précisément de la phytocœnose.

M. CorNIC demande si les travaux italiens ont permis de mettre en corrélation les variations stationnelles et les variations de pertes de production dues au Marssonina, ce point semblant fondamental.

M. Lanier répond que l'article de Castellani et Cellerino (cité en bibliographie) établit des différences de pertes de production selon les clones. Selon la région inventoriée, des différences dues à des variations climatiques apparaissent pour un même clone et sont mises à profit. A propos également du Marssonina brunnea, M. HubERT fait part de ses observations qui montrent que les conditions de milieu, celles qui améliorent la vigueur de l'arbre par exemple, interviennent dans la résistance à certaines maladies. Ainsi le 1214 manifeste une résistance au Marssonina variable selon les stations; les peupleraies bien drainées et travaillées de la vallée de l'Ourcq (Oise) résistent de façon nettement marquée. M. LANIER rappelle que la corrélation vigueur de l'hôte-résistance est très variable selon que l'on est en présence d'un parasite strict ou obligé, dont le type est une Urédinale (Rouille), ou d'un parasite de type saprophyte à tendance parasitaire.

- Dans le premier cas, une règle assez générale veut que la résistance est d'autant meilleure que l'hôte est en moins bonnes conditions de végétation. A la limite, et même lorsque l'hôte est en parfaite condition, peut s'établir une résistance d'un type très particulier, l'hypersensibilité, commandée par des facteurs génétiques et qui fait que, dès l'arrivée du parasite sur l'hôte, se forme une zone nécrotique qui bloque tout développement ultérieur.

- Dans le second cas, auquel se rattachent des parasites du type Marssonina, il apparait au contraire que la résistance est d'autant plus nette que l'hôte est plus vigoureux. M. DupIAs, spécialiste des Rouilles, confirme qu'on a souvent constaté qu'un sujet rendu vigoureux par des apports d'engrais azotés par exemple était plus sensible à l'infection par les Urédinées qu'un sujet poussant dans des conditions défavorables. Les observations de M. DupIAs sur les aires comparées des hôtes et de leurs Rouilles parasites ont montré que l'aire de ces dernières était sous la dépendance de facteurs climatiques (températures extrêmes, hygrométrie...).

Toujours à propos de Marssonina, M. VIART apporte des précisions chiffrées en argent des pertes belges et italiennes. En Belgique, celles-ci se monteraient à 10 millions de dollars US et à 30 millions en Italie. M. LANIER remercie M. VIART de ces précisions, même si leur valeur n'est qu'indicative, car ces chiffres peuvent être utilement comparés avec le coût 
des programmes de recherche devant déboucher sur des méthodes de lutte, comparaison que ne manquent jamais d'établir nos collègues américains.

M. PiCARD demande s'il existe des moyens à employer lors de la plantation pour limiter les dégâts de la Maladie du Rond dans les plantations d'Épicéa effectuées dans des terres agricoles riches.

A cette question d'une portée économique considérable, M. LANIER, relayé par M. Delatour, répond :

Nous avons des recherches en ce sens, qui tendent à établir dès la pépinière et à mettre au point une mycorrhization contrôlée (après démonstration que c'est possible et qu'il y a bien antagonisme entre les champignons mycorrhiziens et les champignons phytopathogènes). Il s'agirait alors d'une "immunisation » des épicéas contre le Fomes.

Mais la biologie du Fomes montre que l'essentiel consiste à éviter l'infection ultérieure par l'intermédiaire des souches d'arbres enlevés en éclaircie. M. Delatour, auteur d'un prochain article sur ce sujet dans la Revue forestière, ajoute que l'importance particulière du Fomes dans les reboisements de terres agricoles est certainement liée aux facteurs du sol : $\mathrm{pH}$ relativement élevé, activité microbiologique différente de celle de la microflore forestière.

M. BALDY demande si l'on a pu chiffrer l'effet des télétoxines provoquées par des champignons ou des insectes piqueurs sur la photosynthèse et sur la productivité du reste de la plante.

M. LANIER ne se cache pas la difficulté à répondre à une telle question : l'effet des toxines est évident dans certains cas, comme celui de la Maladie de l'Orme par exemple, mais il est souvent mal connu et peu apparent dans le domaine des phytopathogènes forestiers.

Il est par ailleurs difficile à différencier des autres effets plus classiques de spoliation ou de troubles du métabolisme causés par la présence de champignons.

La seule formule expérimentale sûre consisterait à isoler les télétoxines en cause et à les inoculer seules à un arbre sain, séparant ainsi leurs effets de tous les autres. 